The Visibility of Information Science and Library Science Research in Bibliometric Mapping of the LIS Field

Author(s): Fredrik Åström

Source: The Library Quarterly, Vol. 80, No. 2 (April 2010), pp. 143-159

Published by: The University of Chicago Press

Stable URL: http://www.jstor.org/stable/10.1086/651005

Accessed: 26/01/2011 19:27

Your use of the JSTOR archive indicates your acceptance of JSTOR's Terms and Conditions of Use, available at http://www.jstor.org/page/info/about/policies/terms.jsp. JSTOR's Terms and Conditions of Use provides, in part, that unless you have obtained prior permission, you may not download an entire issue of a journal or multiple copies of articles, and you may use content in the JSTOR archive only for your personal, non-commercial use.

Please contact the publisher regarding any further use of this work. Publisher contact information may be obtained at http://www.jstor.org/action/showPublisher?publisherCode=ucpress.

Each copy of any part of a JSTOR transmission must contain the same copyright notice that appears on the screen or printed page of such transmission.

JSTOR is a not-for-profit service that helps scholars, researchers, and students discover, use, and build upon a wide range of content in a trusted digital archive. We use information technology and tools to increase productivity and facilitate new forms of scholarship. For more information about JSTOR, please contact support@jstor.org. 


\title{
THE VISIBILITY OF INFORMATION SCIENCE AND LIBRARY SCIENCE RESEARCH IN BIBLIOMETRIC MAPPING OF THE LIS FIELD
}

\author{
Fredrik Åström ${ }^{1}$
}

\begin{abstract}
The relation between information science and library science has been debated for decades, and even attempts at utilizing methods generally acknowledged as robust for the purpose of mapping research fields have yielded results with large variations. Therefore, a set of citation analyses was performed, comparing the results of analyses on information science and library science separately but also as a joint library and information science (LIS) field. Although there are large differences, not the least in the author-level analyses, the patterns in the citation data support the concept of a joint LIS field with information science and library science being the two main subfields; many of the variations in the analyses are caused by the interdisciplinary nature of LIS, reflected in, for example, variations in citation practices in the different subfields.
\end{abstract}

\section{Introduction}

The relationship between library science (LS) and information science (IS) has been debated for decades. The early development of the two fields, together with the influence of the documentation movement, has been discussed by Michael K. Buckland [1], and the benefits of cooperation between the two fields were described by Jesse H. Shera [2] around the time that library and information science departments started appearing. Later contributions in the discussion include, for example, Pertti Vakkari [3] and Peter Ingwersen [4], describing library science (LS) as a subfield within information science (IS), and Tefko Saracevic [5] seeing the two as separate-although closely related-fields. Saracevic's conclusion is interesting, since his description of the intellectual structure of IS draws

1. Lund University Libraries, Head Office, P.O. Box 134, SE-221 00, Lund, Sweden; E-mail fredrik.astrom@lub.lu.se; and University of Technology, Sydney, P.O. Box 123, Broadway, NSW 2007, Australia; E-mail Fredrik.astrom@uts.edu.au.

[Library Quarterly, vol. 80, no. 2, pp. 143-159]

(C) 2010 by The University of Chicago. All rights reserved. 0024-2519/2010/8002-0002\$10.00 
heavily on a bibliometric analysis by Howard D. White and Katherine W. McCain [6] where the intellectual structures of the field are primarily made up of two subfields, informetrics and information retrieval (IR), whereas library science-oriented research is almost nonpresent.

However, according to one of my earlier articles (Åström [7]), the results of White and McCain reflect the selection of journals included in the analysis rather than the structure of library and information science (LIS). Whereas White and McCain [6] analyze documents from eleven journals with a strong emphasis on IS journals, Åström [7] analyzes documents from nine journals, but with a selection including five LS and four IS journals, finding a library science-oriented subfield together with the informetric and the IR subfields. Whether LS is included or not depends not only on journal selection, however. In an article analyzing LIS from different perspectives and using different methods, Félix Moya-Anegón, Víctor Herrero-Solana, and Evaristo Jiménez-Contreras [8] find LS, together with, for example, information management research, being included or excluded depending on what level the co-citation analyses are done.

This raises questions on the perception of library science and information science, especially on the relation between the two and also whether the inclusion of LS research in the cases of Åström [7] and Moya-Anegón, Herrero-Solana, and Jiménez-Contreras [8] depends on whether IS and LS authors and journals actually cite each other or if the inclusion of LS in the maps is solely because of adding the LS journals to the empirical material. To investigate this, this article will produce and compare cocitation maps based on material similar to Åström [7] and Moya-Anegón, Herrero-Solana, and Jiménez-Contreras [8]; to be able to further investigate the IS-LS relation, the material will be examined by analyzing the whole material as well as the information science and library science material separately. And in addition to investigating the structures in the cocitation maps, the distribution of co-citations and the co-occurrence of shared references between IS and LS authors and journals will also be analyzed.

Material and Method

As already noted, when doing these kinds of analyses, the journal selection is of major importance; in doing this analysis, a main task was to identify a selection of IS journals and LS journals. The categorization was based on an analysis of journal titles and the clustering of journals by MoyaAnegón, Herrero-Solana, and Jiménez-Contreras [8]. They based their selection on the Thomson Scientific: Journal Citation Report ranking of information science and library science journals, without making any a 
TABLE 1

JOURNALS FOR ANALYSIS

\begin{tabular}{ll}
\hline \hline IS Journal & \multicolumn{1}{c}{ LS Journal } \\
\hline Information Processing and Management & College E Research Libraries \\
Information Research & Interlending and Document Supply \\
Information Society & Journal of Academic Librarianship \\
Information Systems Journal & Library Quarterly \\
Journal of Documentation & Library Resources and Technical Services \\
Journal of Information Science & Portal-Libraries and the Academy \\
1,696 articles & 1,505 articles \\
54,313 references & 28,137 references \\
\hline
\end{tabular}

NoTE.-IS = information science; LS = library science.

priori distinction between IS and LS journals. Instead, the distinction is the result of their analyses. The title analysis is crude, focusing on whether the journals emphasize "information" or "libraries" in the title. However, when comparing the distinction made in the title analysis and the results in the Moya-Anegón, Herrero-Solana, and Jiménez-Contreras article, the crude title analysis is supported. Drawing upon this, the definition of IS or LS-oriented authors is those authors primarily publishing, or being cited, in LS journals.

Apart from the category selection, an additional criterion for selecting journals was a similar publication frequency among the journals (between four and six issues/year or volume). The reason for this was to be able to work with raw citation and co-citation counts, without journals with substantially higher publication frequencies biasing the distribution of citations. An alternative would be, of course, to use methods for normalization of the data, but the use and usefulness of normalization strategies is debated, not the least in terms of whether or not normalization procedures have any influence on the results or if normalization is of primary importance in relation to what further analyses are being made $[9,10]$. Based on the categorization and the publication frequency criteria, the six highest-ranked IS and LS journals, respectively, in Thomson Scientific: Journal Citation Reports (JCR, 2006 Social Science Edition) were selected for analysis (table 1). All research articles published between 1996 and 2005 in these twelve journals were downloaded using the Thomson Scientific: Web of Science. In total, the data set consists of 3,201 source items citing 82,450 references.

The downloaded data were processed and analyzed using the Bibexcel software [11], a "toolbox" for bibliometricians, making it possible to extract, for example, the "cited references" field from the Web of Science records and to perform a set of various bibliometric analyses. When doing citation analysis, as in this case, there are two basic units involved: the source items, or the citing documents from which the reference lists are 
TABLE 2

Co-Citation Matrix

\begin{tabular}{|c|c|c|c|c|c|c|}
\hline Bates & 0 & & & & & \\
\hline Belkin & 8 & 0 & & & & \\
\hline Cronin & 25 & 49 & 0 & & & \\
\hline Dervin & 30 & 42 & 10 & 0 & & \\
\hline Ellis & 36 & 63 & 28 & 37 & 0 & \\
\hline \multirow[t]{2}{*}{ Ingwersen } & 33 & 53 & 3 & 60 & 58 & 0 \\
\hline & Bates & Belkin & Cronin & Dervin & Ellis & Ingwersen \\
\hline
\end{tabular}

collected, and the cited references, or the documents found in the reference lists. Also, the analyses can be performed on different levels of aggregation; for example, if we are analyzing the documents per se, or the authors-that is, the collected works of individual authors, or the journals-for example, the citations to all articles in one journal. Based on this, we can analyze frequencies, co-occurrences of properties, and to what extent documents share certain properties. In this case, the focus will be on author co-citation analyses (ACA) $[12,13]$, that is, to what extent the aggregated works of authors are cited together, and journal co-citation analyses (JCA) [14], the extent to which the collected number of articles in journals are being cited together.

The co-citation analysis takes its starting point in the cited references, looking for authors or journals that appear together in the reference lists of the downloaded articles, assuming that two texts being cited together have some topical or intellectual similarity. And the more times, for example, an author is being co-cited with another author, the stronger we assume that the connection-or co-citation strength-is between them [12, 13]. This makes it possible for us to see authors with high co-citation strengths forming areas of shared research interests and also to see to how different research areas relate to each other. Thus, in this article, we are looking for to what extent IS and LS authors and journals are cited together.

To be able to make a graphic representation of these relations, the first step of the analytical work was to select which units to analyze (i.e., the authors or journals to be included in the analysis), which was done by ranking authors/journals according to the extent to which they have been cited together and selecting the ones with the highest citation frequencies. The selected authors/journals were then organized in a co-citation matrix, showing how many times each of the selected items had been cited together (table 2).

In the next step, the values in the matrix were used as proximity measures for the MDS Alscal scaling algorithm, an algorithm for representing multidimensional data in two- or three-dimensional spaces by producing a scatter 
plot where co-cited pairs are placed closer or further away from each other depending on how often they are co-cited, that is, the co-citation strength. Since the proximity measures in the multidimensional data are manipulated to reduce dimensions in the visual representation, a best-fit measurement called "stress value" is calculated to see to what extent the twodimensional representation deviates from the co-citation relations in the matrix, where a stress value below 0.2 is considered acceptable [15].

To inquire further into the relation between IS and LS-and to investigate whether there are any actual relations between the two fields or if the inclusion of LS in previous mapping exercises is artificial and depends on the inclusion of LS journals- the distribution of co-citations and shared reference percentages were calculated. Shared reference, or bibliographic coupling, analysis is similar to co-citation analysis, but instead of connecting references appearing in the same reference lists, it connects documents sharing the same references [16]. In the case of this article, this means that the analyses investigates to what extent IS and LS authors and journals are referring to the same literature. As well as with the maps, the analyses were performed on both the author and journal levels. The shared reference analysis was performed on the whole document set by dividing the number of authors or journals cited in both IS and LS articles by the total number of cited authors or journals. The distribution of citations between IS and LS was calculated on a selection of the most-cited authors/journals in the two fields, respectively, by analyzing to what extent references were co-cited with exclusively IS, LS, or with both IS and LS, references. For instance, the fifteen most-cited IS authors were selected, and for each of the individual authors, the authors they were co-cited with were classified as either an IS or LS author. Based on this, we can see to what extent IS authors are co-cited with IS or LS authors and, on an aggregated scale, to what extent co-citations are distributed within IS or LS or between the two.

Results

Author-Level Analyses

The first analysis is an author co-citation analysis based on the whole material (IS+LS+LIS), including the forty-nine author names receiving fifty or more citations. Out of these, 904 unique co-citation pairs were identified, and, based on the co-citation strength (number of co-citations), the authors were placed in relation to each other using the MDS Alscal algorithm (fig. 1). To further enhance the structures of the MDS map, an alternative to the MDS analysis was used: a cluster analysis to reveal structures in the matrix by dividing data sets into smaller subsets and thus classifying objects into groups on the basis of common traits. In this case, a clustering routine 


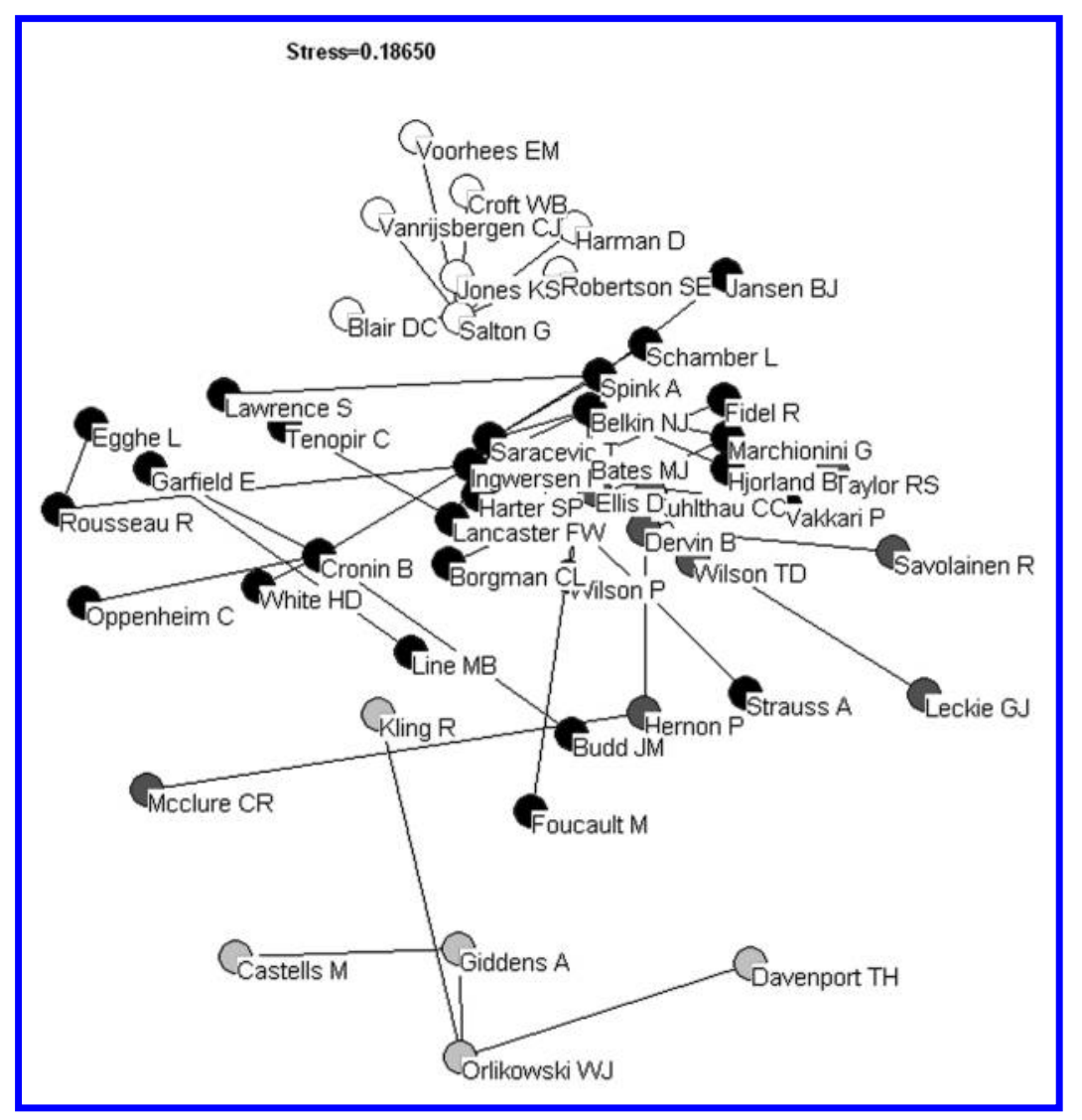

FIG. 1.-Author co-citation analysis on IS, LS, and LIS journals, including the forty-nine most-cited authors.

suggested by Olle Persson [17] was used, and the results form the basis of the links between the authors in the ACA. Instead of using the co-cited pairs as the basic unit of analysis, this method focused on grouping cocited pairs sharing at least one unit, thus the pairs A-B and B-C form a cluster, whereas the pairs A-B and C-D do not. Not only does this mean that we get clearer and more easily interpretable structures but also structures that are based on de facto citation relations between authors or journals and not on a statistical reduction of dimensions, serving to counteract the relatively high stress value.

Thus, the resulting map represents not only the relative proximity between co-cited authors through the MDS analysis but also the structure revealed by the cluster analysis, represented by the links between cited 
authors within the different clusters. The main structure of the map is largely familiar, similar to earlier maps [6-8]. On the top half, we find the bibliometric research on the left side, represented by, for example, Leo Egghe, Eugene Garfield, and Howard D. White; the systems-oriented IR on top with, for example, Cornelis Joost van Rijsbergen and Gerard Salton; and at the right side, a movement from information searching and seeking to needs and uses research represented by, for example, Linda Schamber, Peter Ingwersen, and Reijo Savolainen. However, as opposed to the White and McCain analysis [6], underneath the bibliometrics and informationseeking and use areas, we find an area with primarily LS-oriented researchers, such as Charles R. McClure, John M. Budd, and Gloria J. Leckie, which was also reflected in Åström [7]. A new addition to the mapping of LIS is the area at the very bottom, reflecting an increasing interest in general theorists such as Manuel Castells, Michel Foucault, and Anthony Giddens, authors not being part of the LIS field but having a significant enough impact on LIS research over the past ten years to be seen in an unrestricted citation analysis.

When doing the same kind of analysis on the forty-two top-cited authors in the information science journals, the results are almost identical, with the exception of an exclusion of the LS area and a lower stress value, 0.15 , as opposed to a high 0.19. It is also noticeable how authors common to the IS and LS maps are exclusively IS authors also cited in LS articles, whereas the IS+LS+LIS map includes authors primarily related to the LS area, indicating a low degree of connection between LS and IS as seen by the citing IS scholars. This supports the assumption of MoyaAnegón, Herrero-Solana, and Jiménez-Contreras [8] that LS authors to a lesser extent reach citation frequencies high enough to meet the threshold for inclusion in an LIS map, and it can also be noted that the distribution of citations is less skewed among LS-cited authors than in-between IS authors. This is reflected in the threshold value for inclusion in the LS ACA map being only twenty, as well as the low 268 unique pairs formed, while still having a higher stress value (fig. 2). However, it should also be noted that LS articles have fewer references: whereas IS articles have an average of thirty-two references per article, the corresponding figures for LS articles is nineteen, as can be seen in table 1 .

The structure of the LS map is not as clear-cut as the map covering all, or just the IS, authors. Whereas the LIS map showed a number of distinct research areas, the LS map has very few traces of distinct groups of authors. However, as opposed to the IS map, in the LS map we find cited authors with strong relations to both IS and LS research, such as F. Wilfred Lancaster. This would indicate a stronger relation between IS and LS, at least as seen through the eyes of citing LS authors. An interesting aspect is how neither of the maps presented so far makes a cluster-based distinction 


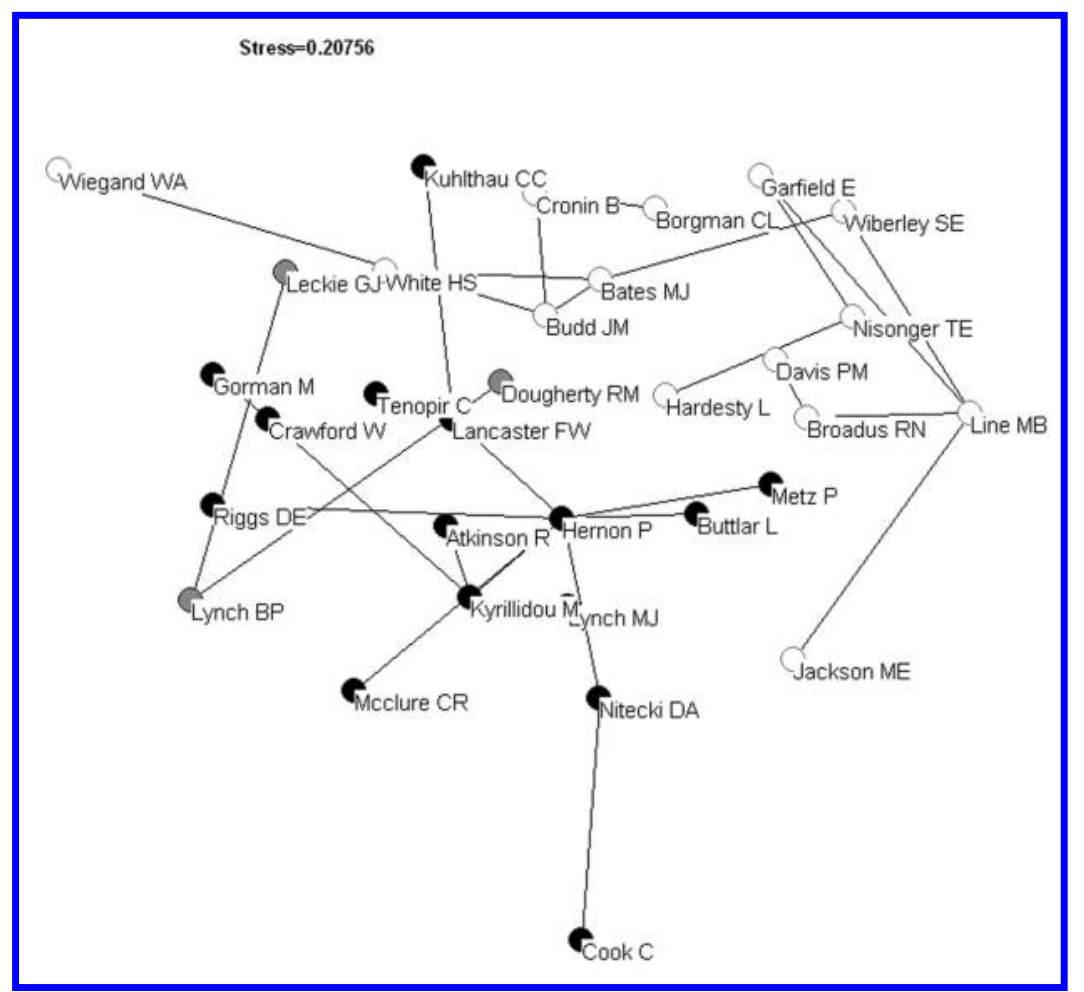

FIG. 2.-Author co-citation analysis on LS journals, including the thirty-two most-cited authors.

between IS and LS authors. The only time the cluster analysis yields such a result is when making a citations among documents (CAD) ACA analysis on the IS+LS+LIS journals [18]. The CAD analysis enforces a restriction on which citations are counted by limiting the analysis to citations within the set of citing documents. Thus, if we would be analyzing citations from articles in, for example, the Journal of Documentation published from 1990 to 2000 , we would only study the citations coming from the same journal within the same time frame. This is done by extracting a search key from the source documents containing author, publication year, volume number, and the beginning page number of each article. Based on this, the references lists of the articles are searched, and the references that are also part of the set of source documents are extracted for further analysis. The idea is, on the one hand, to avoid topic drift through citations to research outside the field whose journals are analyzed and, on the other, to be able to focus on contemporary research. Within the document set, 3.369 citations went to other source items, identified by the aforementioned search 


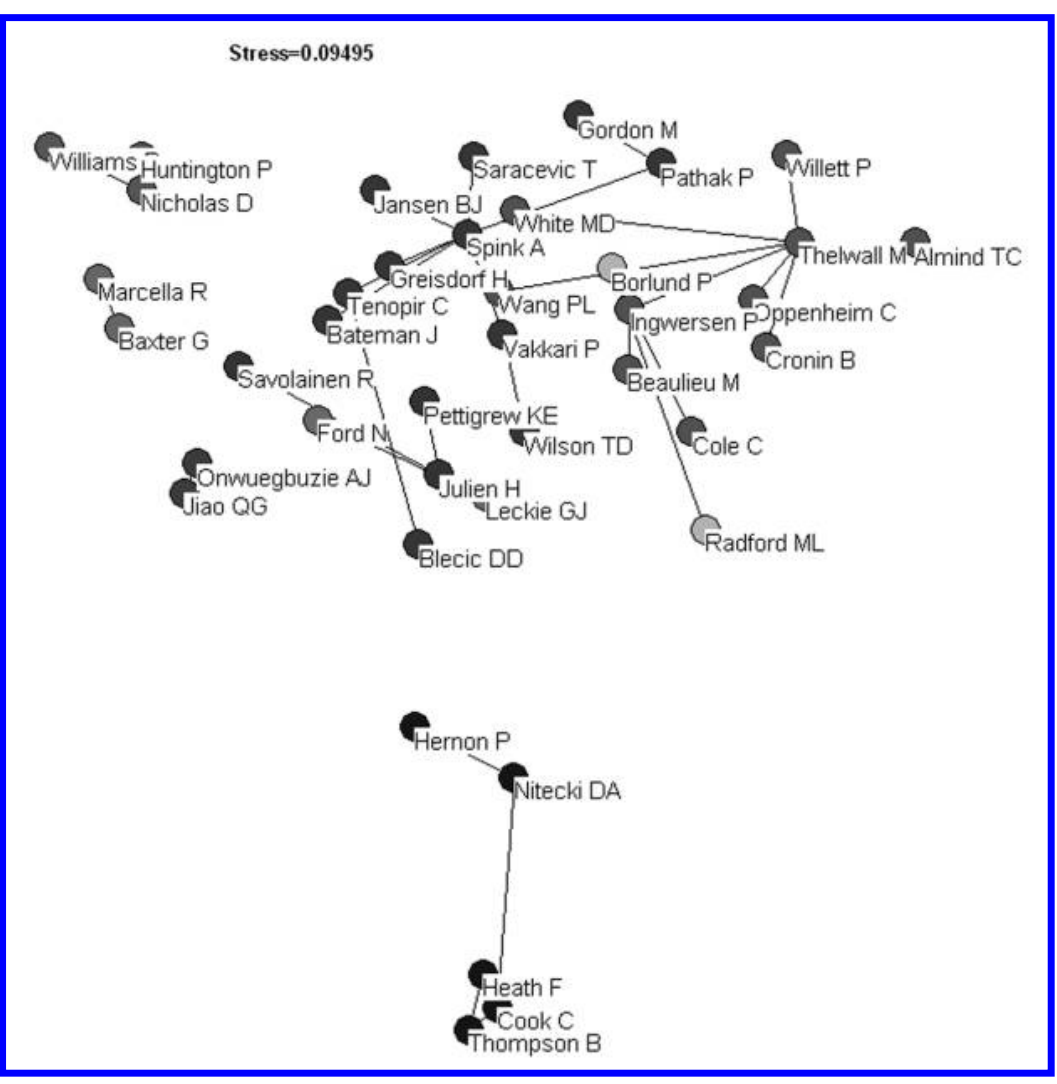

FIG. 3.-Author-level citation among documents analysis on IS, LS, and LIS journals, including the forty most-cited authors.

key, and out of the 1.716 unique cited authors, identified by ranking authors according to number of citations per author, the ones receiving forty or more citations were chosen for analysis where they formed 361 unique pairs (fig. 3).

As opposed to the non-CAD ACA analysis, there are only two LS authors included in clusters also featuring IS authors, while the rest of the LS authors are gathered in two separate clusters, meaning that, among the source documents, the distinction between IS and LS authors is more noticeable than in the unrestricted analysis. In all, the MDS-generated maps do not give a conclusive result on the connections between IS and LS, although there is a certain level of overlap between the two areas.

To investigate the IS-LS overlap further, the number of shared references between IS and LS, as well as the distribution of co-citations between the 
TABLE 3

Aggregated Distribution of Co-citations between the Fifteen Most-Cited IS And LS Authors, Respectively (261 Unique Pairs)

\begin{tabular}{lcc}
\hline \hline & \multicolumn{2}{c}{ Co-citations } \\
\cline { 2 - 3 } Co-Cited Area $(N=$ & Co-citation Frequency $(\%)$ & $\begin{array}{c}\text { Discrete Amount of } \\
\text { Co-cited Pairs }(\%)\end{array}$ \\
Co-Citations/Pairs $)$ & 77 & 39 \\
\hline IS $(N=2.347 / 101)$ & 6 & 21 \\
LS $(N=166 / 56)$ & 17 & 40 \\
IS + LS $(N=521 / 104)$ & & \\
\hline
\end{tabular}

NoTE - IS = information science; LS = library science.

two areas, was investigated further. When comparing cited authors in the IS and LS journal articles, respectively, to all cited authors in the document set, only 6 percent of the cited authors $(N=4,045)$ are cited by both IS and LS journal articles, making up 8 percent of the total citation frequency $(N=5,685)$. The strong division between the two areas suggested by these figures is even more noticeable when looking at the aggregated distribution of co-citations among the fifteen most-cited authors in the citations among documents' ACA analysis, where only 5 percent $(N=104)$ of the co-cited pairs, or a mere 1 percent of the total co-citation frequency $(N=521)$, show co-citation links between IS and LS authors. However, removing the limitations of the CAD analysis and including all works-cited authors in the source items dramatically changes the distribution of co-citations (table $3)$.

Here, IS authors co-cited with other IS authors make up 39 percent of the co-cited pairs, while 40 percent are pairs where one author is identified as an IS author and the other an LS author, that is, a much stronger connection between the two areas. It should however be noticed that the correlation between co-citation frequency distribution and the number of cited pairs is much lower when looking at co-citation distribution in the unrestricted analysis, although this could be explained by the higher number of citations in the IS articles (table 1). When looking at the co-citedness of individual authors, it also becomes apparent that there are large differences, both in between authors within the areas and how authors are co-cited across the two areas. Whereas 50 percent of the cited LS authors are co-cited with IS authors in more than 50 percent of the co-cited pairs they are included in, the reverse numbers are 17 percent IS authors cocited with LS authors in more than 40 percent of "their" pairs, and none above 50 percent. Not only are LS authors co-cited with IS authors to a larger extent than vice versa but the number of authors with very high intra-area distributions-8 percent and more-is higher in the IS area, both in terms of number of authors and co-citations. 


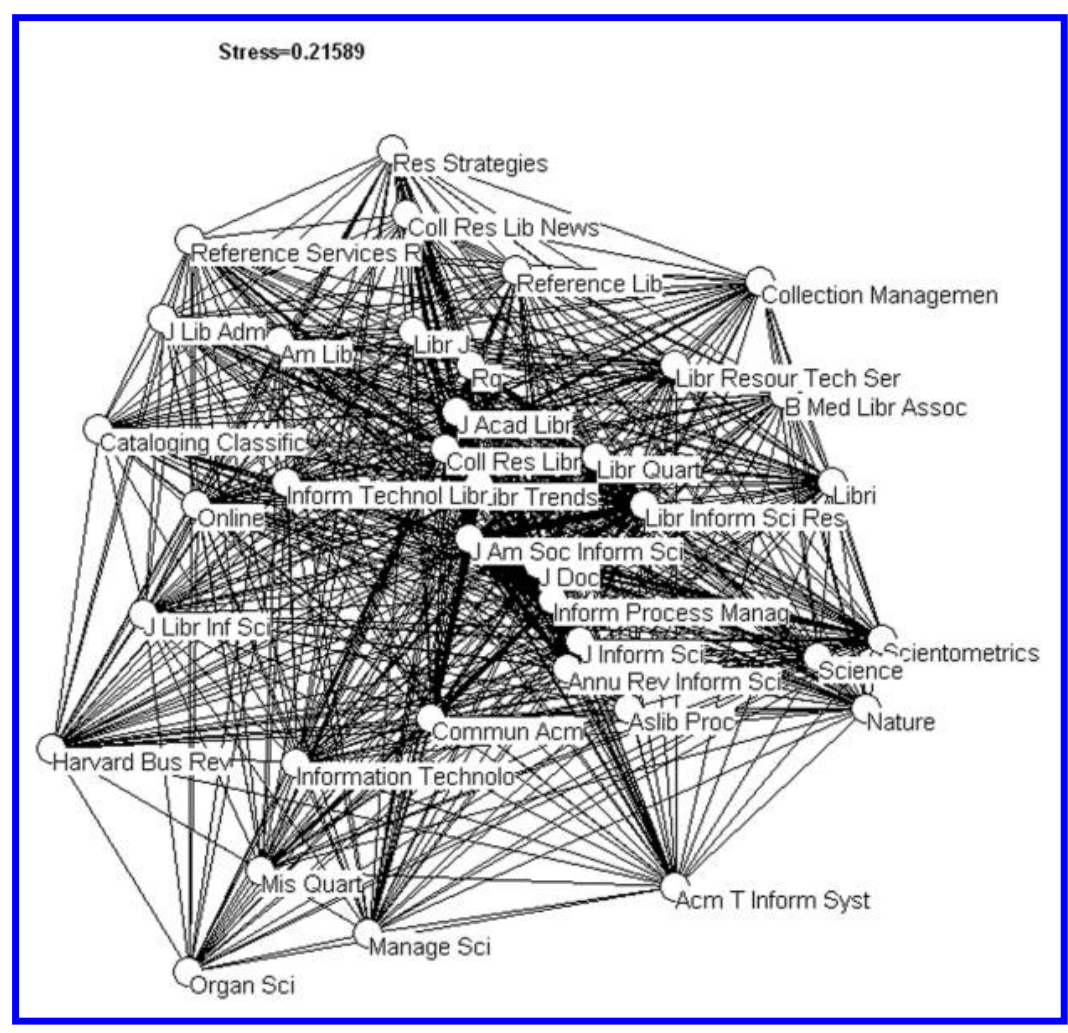

FIG. 4.- Journal co-citation analysis on IS, LS, and LIS journals, including the thirty-seven most-cited journals.

Journal-Level Analyses

As noted in the introduction, whether the co-citation analyses are done on the author or journal level has a big impact on how LIS is represented in informetric mapping [8]. As in the Moya-Anegón, Herrero-Solana, and Jiménez-Contreras study, a journal co-citation analysis was performed, to start with, on the whole material (IS+LS+LIS). The analysis included thirty-seven journals receiving one hundred or more citations, and out of these, 628 unique co-citation pairs were identified and mapped in relation to each other based on the co-citation strength (fig. 4).

Although a high stress value indicates that the results should be viewed with caution, the results are similar to Moya-Anegón, Herrero-Solana, and Jiménez-Contreras [8], with a collection of LS-oriented journals on the top, IS journals in the center and on the lower right side, and a management-oriented field at the bottom left. The only substantial difference is the lesser presence of science studies literature in this analysis. Another 


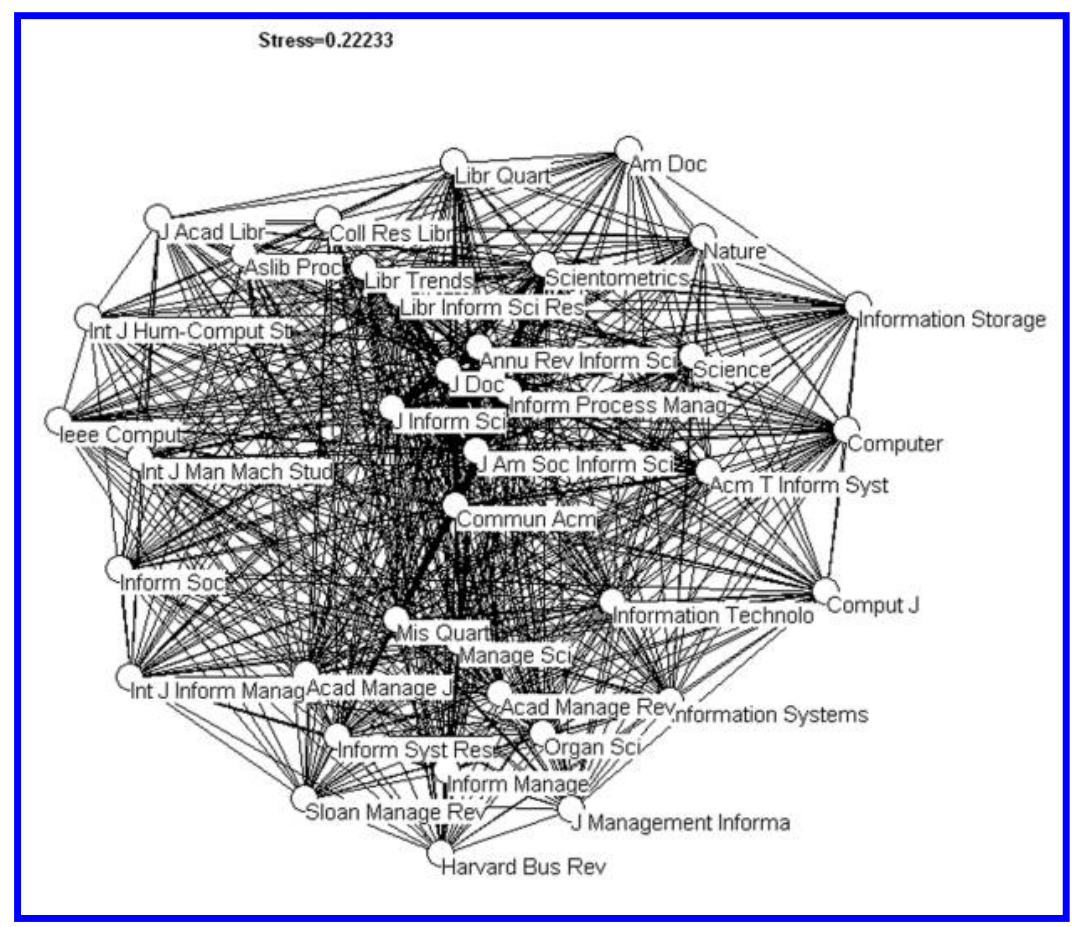

Fig. 5.-Journal co-citation analysis on IS journals, including the thirty-seven most-cited journals.

difference is how the structure of the map is less distinct: the boundaries between areas and the grouping of journals are not as clear as in the ACA, which is also indicated by the high stress value and the lack of clusters forming when applying the clustering routine. However, whereas the ACA based on IS journal articles revealed an introspective focus on IS authorswith the exception of the group of general theorists at the bottom-the JCA IS journals are more directed outward. When analyzing the thirtyseven IS journals receiving fifty citations or more-and the 611 unique pairs formed in between these thirty-seven journals-we find a distinct core of IS journals in the center (fig. 5). Surrounding the center, we find a group of LS journals on top, a large group of management studies journals both at the bottom and on both sides, and also a number of computer science journals. It is, of course, interesting to note the influence of management and computer studies journals on information science research, but, more important, this map suggests that the appearance of LS journals on the all-journals maps and in the Moya-Anegón, Herrero-Solana, and 


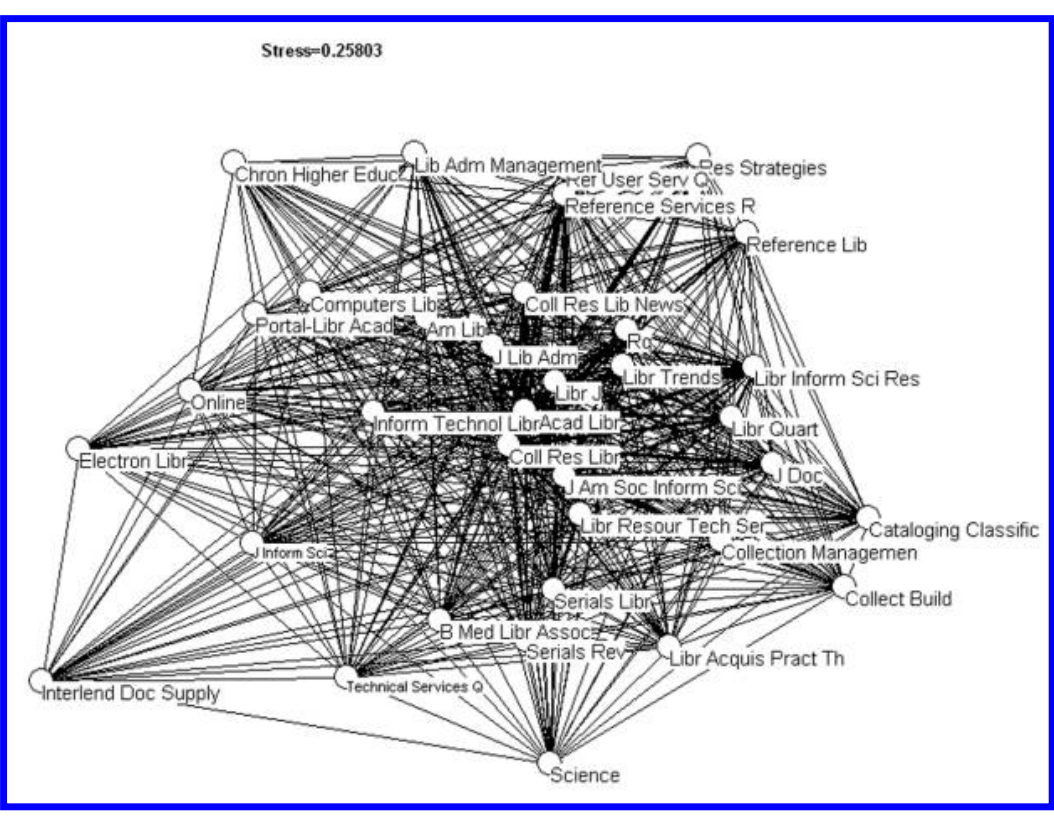

Fig. 6.-Journal co-citation analysis on LS journals, including the thirty-five most-cited journals.

Jiménez-Contreras [8] analysis is based on an existing connection between the two areas and not just the adding of LS journals to the data material.

While the JCA maps of IS show a significant representation of LS journals, the JCA on LS does not show the same connection between the two areas (fig. 6). When analyzing the thirty-five journals receiving forty or more citations in LS journals, 567 unique pairs are found, but among the thirty-five journals, few of those are IS journals and also few are representing other research areas. Instead, the core of general LS journals in the center is surrounded primarily by LS journals representing different specializations, such as library administration, reference services, or collection management. Whereas the ACA revealed a structure where LS journal articles cited IS authors, rather than the other way around, the case is reversed when looking at the JCA maps of IS and LS, respectively, with a larger presence of LS journals cited in IS articles rather than vice versa.

The general view of IS and LS being closer to each other in the JCA is supported both by looking at the extent to which IS and LS journal articles are citing the same journals and by the distribution of co-citations. While the number of common citations to authors' in-between IS and LS journal 


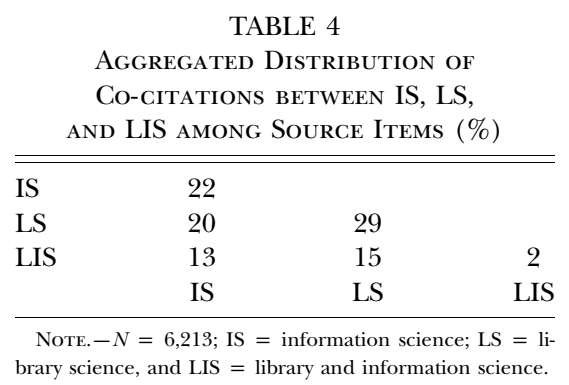

articles was only 8 percent, the corresponding percentage on journal level is 55 percent. And while 77 percent of the author co-citations were between IS-IS authors and 17 percent between IS-LS authors, the journal co-citations are much more evenly distributed (table 4).

As with the ACA, there are large variations in how individual journals are being co-cited with IS and LS journals. Among the IS journals, cocitations with other IS journals range between 29 percent and 88 percent, with a median of 55 percent, while the corresponding figures for LS-LS journal co-citation is from 44 percent to 77 percent, with a median of 70 percent. As indicated by the LS map, the LS journals are to a higher extent co-cited with other LS journals, while IS journals have a more even distribution of co-citations in between IS and LS journals.

\section{Discussion}

The relation between information science and library science has been debated for decades, and even empirical investigations into the LIS journal literature have provided varying results. In White and McCain's [6] article in the Journal of the American Society for Information Science, the library science field is almost nonexistent, providing an argument for Saracevic's [5] division between IS and LS, while for Moya-Anegón, Herrero-Solana, and Jiménez-Contreras [8], the inclusion or exclusion of LS in a wider LIS/IS field depends on the level of analysis and the generally lower citation frequencies for LS authors. To see if the inclusion of LS research in, for example, Moya-Anegón, Herrero-Solana, and Jiménez-Contreras's [8] JCA is because of de facto citation relationships between IS and LS or because of LS journal articles being added to the empirical material, the mapping exercises on IS and LS was revisited, and further investigations into individual and general citation relationships between the two areas were also conducted.

The conclusion that LS research is excluded because of low citation frequencies among LS authors can to some extent be confirmed by the 
analyses presented here. The threshold value for being included in the LS map is twenty citations, while the corresponding value for the IS+LS+LIS analysis is fifty citations or more; the highest-cited LS author received eighty citations, while the corresponding number among the IS journals is 260 . However, when looking at the distribution of co-citations between IS and LS authors, as well as to what extent IS and LS journal articles share references, there are few connections between IS and LS authors. The exception is the distribution of discrete numbers of authors, where 40 percent of the co-citations are between IS and LS authors, while there is a 17 percent distribution of co-citations between IS and LS when taking co-citation frequency into account. At the same time, it is interesting to note the higher presence of IS authors cited in LS articles, which would support the notion of IS being the more general field and LS a subfield to IS, as suggested by, for example, Vakkari [3] and Ingwersen [4].

This division between IS and LS on the author level is further emphasized by the citations among documents (CAD) ACA analysis of IS+LS+LIS journal articles, where the majority of included LS authors are distinctly separated both in terms of position on the map as well as in the cluster analysis. It is also interesting to note that many of the author names being common to the unrestricted IS, LS, and all-journal analyses are not found in the CAD analysis. This suggests that many of the highly cited authors active in both IS and LS research, as well as being highly influential, are authors who have been active over many decades but whose publication activities have dwindled and/or whose newer publications receive fewer citations. At the same time, it is also important to acknowledge that LS authors are not only cited less but their articles also contains fewer references, meaning there is a difference in citation praxis in LS research, making it harder for individual scholars to amass larger numbers of citations.

Although the lower citation frequency of LS authors does not seem to be a primary explanation for the exclusion of LS in ACA maps, there are still good arguments against a strong division between IS and LS. When looking at the results of the JCAs, the connection between IS and LS is much stronger both in the maps and in the further analyses into co-citation distribution and shared references between IS and LS journal articles. The two areas are both being co-cited together to a larger extent as well as citing the same journals from both IS and LS. A somewhat peculiar result is how, while on the author level LS journal articles seem to be citing IS authors more than vice versa, in the journal-level analysis the case is reversed, with IS journal articles citing LS journals to a larger extent than LS articles are citing IS journals. One explanation for this might be that not only are general issues to a larger extent approached in IS journals, while LS journals are more specialized on specific issues, but the references to general issues presented in IS journals are also going to a relatively 
small number of IS scholars who could be seen as "citation classics" representing different IS research areas.

One final issue that needs to be addressed is the large difference between percentage of distribution when looking at numbers of co-citation frequencies/shared references and numbers of pairs, especially in terms of author co-citations. Although there seem to be few connections between IS and LS on the author level, it also seems that most of the connections actually being there are primarily hidden in a "long tail" of authors receiving very few citations at the same time that there is not that long a "tail" in terms of journals. So even though there are significant differences in terms of numbers of citations to journals in IS and LS journal articles, respectively, this is compensated by the difference between the total number of cited journals in relation to the number of cited authors.

\section{Conclusions}

Based on citation data from research articles published in scholarly LIS journals, there is evidence for relating information science and library science together in a joint field of LIS, with IS and LS being the two main subfields. A further division into more specialized research areas can also be found when analyzing IS citation data, whereas LS research areas are less distinctly visible in citation analyses but can be traced through specialized foci of different scholarly journals. There are, however, big differences between the two subfields, not the least visible in the author-level citation analyses, where the connections are quite weak. But rather than seeing them as reflecting a division of IS and LS, they can be seen as strong indicators on the interdisciplinary nature of LIS, not only in the wide range of different research interests visible in the analyses but also in variations in, for example, citation practices, reflecting general differences in the organization of IS and LS research, respectively.

\section{REFERENCES}

$\rightarrow$ 1. Buckland, Michael K. "Documentation, Information Science, and Library Science in the U.S.A." Information Processing and Management 32 (1996): 63-76.

2. Shera, Jesse H. "Of Librarianship, Documentation and Information Science." UNESCO Bulletin for Libraries 22 (March-April 1968): 58-65.

3. Vakkari, Pertti. "Library and Information Science: Its Content and Scope." In Advances in Librarianship: Volume 18, edited by I. P. Godden, pp. 1-55. San Diego, CA: Academic Press, 1994.

$\rightarrow$ 4. Ingwersen, Peter. "Information and Information Science in Context." Libri 42 (1992): 99-135. 


\section{BIBLIOMETRIC MAPPING OF THE LIS FIELD}

$\rightarrow$ 5. Saracevic, Tefko. "Information Science." Journal of the American Society for Information Science 50 (1999): 1051-63.

6. White, Howard D., and Katherine W. McCain. "Visualizing a Discipline: An Author Cocitation Analysis of Information Science, 1972-1995." Journal of the American Society for Information Science 49 (1998): 327-55.

7. Åström, Fredrik. "Visualizing Library and Information Science Concept Spaces through Keyword and Citation Based Maps and Clusters." In Emerging Frameworks and Methods: CoLIS4, edited by Harry Bruce, Raya Fidel, Peter Ingwersen, and Pertti Vakkari, pp. 185-97. Santa Barbara, CA: Libraries Unlimited, 2002.

$\rightarrow$ 8. Moya-Anegón, Félix; Herrero-Solana, Víctor; and Jiménez-Contreras, Evaristo. "A Connectionist and Multivariate Approach to Science Maps: The SOM, Clustering and MDS Applied to Library and Information Science Research.” Journal of Information Science 32 (2006): 63-77.

$\rightarrow$ 9. Schneider, Jesper W., and Pia Borlund. "Matrix Comparison, Part 1: Motivation and Important Issues for Measuring the Resemblance between Proximity Measures or Ordination Results." Journal of the American Society for Information Science and Technology 58 (2007): 1586-95.

$\rightarrow$ 10. White, Howard D. "Author Cocitation Analysis and Pearson's r." Journal of the American Society for Information Science and Technology 54 (2003): 1250-59.

11. Persson, Olle; Danell, Rickard; and Schneider, Jesper W. "How to Use Bibexcel for Various Types of Bibliometric Analyses." In Celebrating Scholarly Communication Studies: A Festschrift for Olle Persson at His 60th Birthday, edited by Fredrik Åström, Rickard Danell, Birger Larsen, and Jesper W. Schneider, pp. 9-24. Special volume of the e-zine of the ISSI, vol. 05-S, June 2009. http://www.issi-society.info/ollepersson60/.

$\rightarrow$ 12. White, Howard D., and Belver C. Griffith. "Author Co-citation: A Literature Measure of Intellectual Structure." Journal of the American Society for Information Science 32 (1981): $163-71$.

13. McCain, Katherine W. "Cocited Author Mapping as a Valid Representation of Intellectual Structure." Journal of the American Society for Information Science 37 (1986): 111-22.

$\rightarrow$ 14. McCain, Katherine W. "Mapping Economics through the Journal Literature: An Experiment in Journal Cocitation Analysis.” Journal of the American Society for Information Science 42 (1991): 290-96.

$\rightarrow$ 15. McCain, Katherine W. "Mapping Authors in Intellectual Space: A Technical Overview." Journal of the American Society for Information Science 41 (1990): 433-43.

$\rightarrow$ 16. Kessler, M. "Bibliographic Coupling between Scientific Papers." American Documentation 14 (1963): 10-25.

$\rightarrow$ 17. Persson, Olle. "The Intellectual Base and Research Fronts of JASIS 1986-1990." Journal of the American Society for Information Science 45 (1994): 31-38.

$\rightarrow$ 18. Persson, Olle. "All Author Co-citations versus First Author Co-citations.” Scientometrics 50 (2001): 339-44. 